\title{
Are Prenatal Ultrasounds Safe for the Developing Brain?
}

\author{
A review of: Ang ES Jr, Gluncic V, Duque A, Schafer ME, Rakic P 2006 Prenatal exposure to ultrasound waves \\ impacts neuronal migration in mice. Proc Natl Acad Sci U S A 103:12903-12910
}

$\mathrm{M}$ onitoring the fetus throughout pregnancy has become a major task of modern obstetrical care and ultrasonography, first applied 30 y ago, has revolutionized the possibility of following fetal well-being and of screening fetal organ abnormalities with congenital malformations (e.g., anencephaly, holoprosencephaly, spina bifida, neck and chest malformations such as major congenital heart disease, abdominal malformations such as megabladder or polycystic kidneys, limb defects tumors or structural indications of chromosomal abnormalities) which help in counselling parents. The development of endovaginal probes allowed visualization of early pregnancy as early as 10-14 weeks. More recently, the development of 3D and 4D ultrasound has allowed the physician to assess facial malformations such as cleft palate, but has also moved the technique into public interest for the beautiful "picture in the womb" potential of the technique.

"At Womb with a View, you can spend time with your developing baby. See her move, watch him play. There is no time more precious and cherished during pregnancy than the time that the mother-to-be gets to spend looking at her baby through the miracle of ultrasound. Using state-of-the-art 3D ultrasound technology, we offer expectant mothers the time and opportunity to view the developing baby. This is your time! No frightening measurements. No full-bladder. Just you, your baby and your guests. Womb with a View provides expectant mothers the opportunity to spend quality time with the new baby in a mother-centered environment. We welcome family and friends to join our celebration.

\section{Pierre Gressens \\ Petra S. HüPpI}

http://www.wombwithaview.com/"

In this success story of fetal ultrasonography, the scientific community is alerted by an interesting study conducted by universally-known neuroscientist Pasko Rakic and his team showing that exposure to ultrasonography during neuronal migration leads to migrational arrest in the young rodent brain (1).

The authors exposed pregnant mice to ultrasound waves produced by an ultrasound system routinely used in human medical clinics. The exposure started on embryonic day 16 and lasted for different periods, ranging from $1 \times$ $5 \mathrm{~min}$ to $12 \times 35 \mathrm{~min}$ (with a 4 to $12 \mathrm{~h}$ periods separating the different sessions of ultrasound in cases of multiple exposures). When animals were killed on postnatal day 10 , they did not observe any significant effect on brain size or on gross brain cytoarchitectony.

In addition, in the same animals, the authors injected once on embryonic day 16 bromodeoxyuridine (BrdU), a thymidine analogue incorporated into DNA during $\mathrm{S}$ phase of the mitotoic cells. This allows labelling of a cohort of neuronal precursors which are dividing at that time and which will migrate predominantly to superficial neocortical layers. On postnatal day 10, neurons which had incorporated BrdU were immunolabelled and their distribution along the different cortical layers and underlying white matter was defined, allowing determination of dispersion differences between controls and animals exposed in utero to ultrasound waves. In animals exposed to ultrasound waves for a total duration of $30 \mathrm{~min}$ or more, they observed a significant and dose-dependent increase in dispersion of neurons (from 9\% dispersion difference in the group exposed to $30 \mathrm{~min}$ to $19 \%$ in the group exposed to $420 \mathrm{~min}$ of ultrasound waves), meaning that more neurons failed to migrate in the superficial neocortical layers and remained blocked in the deep cortical layers or even in the underlying white matter. The magnitude of dispersion of labelled neurons was variable, but increased systematically with duration of exposure to ultrasound waves.

Of note, no pups survived when exposed to $600 \mathrm{~min}$ of ultrasound waves and several exposed to $420 \mathrm{~min}$ were cannibalized by the mothers at birth. This suggests that prolonged exposure to ultrasound waves is a stressful event. The present study did not permit the total elimination of the potential role of stress on migration dispersion in the 420-min exposure group. The rat pups of mothers that were exposed to the ultrasound probe for $420 \mathrm{~min}$, but without US waves (US machine switched off), showed a dispersion of $12 \%$ of neurons, which again was significantly higher than the approx. $6 \%$ of dispersed neurons found in normal control pups. No hormonal measurements were made in the pregnant mice during these procedures, so assessment of neuroendocrine modification due to stress hormones cannot be evaluated. It is well known that exposure to corticosteroids during critical periods of brain development alter neuronal progenitor cell survival and proliferation and differentiation (2) and might therefore have contributed to the findings of this study. Interestingly, preterm in- 
fants with intrauterine growth retardation due to placental insufficiency, often followed up with frequent US measurements between 20 and 30 weeks gestation, have been shown to have a $20-$ $30 \%$ reduction in overall cortical volume at birth and at follow-up in the newborn period (3). Preterm infants treated with corticosteroids showed a $35 \%$ reduction of cortical volume compared with nontreated preterm infants (4). No correlative studies exist that would link these observations in human infants to increased neuronal dispersion as observed in the animal model. As mentioned above, pups were killed at postnatal day 10. Although this is at some distance from the end of normal migration, in animals exposed to ultrasound waves this time point does not allow clear discrimination between a definitive arrest of migration of neurons and an important delay in migration.

Translation from small rodents to the human situation is always a challenge as, beyond the conservation of basic mechanisms of brain development, several differences are obvious. As far as neuronal migration for the neocortex is concerned, it occurs in mice mostly between embryonic days 11 and 19 while it extends over several weeks (roughly between 6 to 24 gestational weeks) in humans (5). In this context, a 420-min exposure to ultrasound waves represents more than $3 \%$ of the total period of neuronal migration. In humans, $3 \%$ of the migration period would correspond roughly to $90 \mathrm{~h}$. On the other hand, the migration path in humans is much longer than in mice, which might make migrating neurons more susceptible to ultrasound waves (6). As suggested by the authors themselves, similar studies in nonhuman primates will be necessary to address these key questions.

In terms of the underlying mechanisms, ultrasound waves might affect cell motility through radiation forces and microstreaming of low-viscosity fluids of the extracellular space, leading to disturbances of membranemediated cell-to-cell attachments and nuclear translocation.

With regard to exposure to US waves, the prenatal US assessments in a normal pregnancy are indeed made with $5 \mathrm{MHz}$ probes and do not last much longer than $30 \mathrm{~min}$ and are made for the first time between 16 and 20 weeks. This procedure has been shown in a large trial to reduce perinatal mortality, increase malformation detection rate and decrease healthcare costs (7). Both routine and repeated ultrasound in later pregnancy (after 24 weeks) and routine Doppler ultrasound measurements in unselected and low-risk pregnancies do not confer benefit for mother or baby $(8,9)$. In view of the above, one can conclude that fetal ultrasound assessment should be performed upon clear clinical indication following evidence-based medical decisions and the advertised view into the womb might be more stressful and potentially harmful for the fetus than the desired quality time with your baby.

\section{REFERENCES}

1. Ang ES Jr, Gluncic V, Duque A, Schafer ME, Rakic P 2006 Prenatal exposure to ultrasound waves impacts neuronal migration in mice. Proc Natl Acad Sci USA 103:12903-12910

2. Wong EY, Herbert J 2006 Raised circulating corticosterone inhibits neuronal differentiation of progen- itor cells in the adult hippocampus. Neuroscience 137:83-92

3. Tolsa CB, Zimine S, Warfield SK, Freschi M, Sancho RA, Lazeyras F, Hanquinet S, Pfizenmaier M, Hüppi PS 2004 Early alteration of structural and functional brain development in premature infants born with intrauterine growth restriction. Pediatr Res 56:132-138

4. Murphy BP, Inder TE, Hüppi PS, Warfield S, Zientara GP, Kikinis R, Jolesz FA, Volpe JJ 2001 Impaired cerebral cortical gray matter growth following treatment with dexamethasone for neonatal chronic lung disease. Pediatrics 107:217-221

5. Gressens P, Huppi PS 2006 Normal and abnormal brain development. In: Martin RJ, Fanaroff AA, Walsh MC (eds) Neonatal-Perinatal Medicine. Mosby, Philadelphia, pp 883-909.

6. Gressens P 2000 Mechanisms and disturbances of neuronal migration. Pediatr Res 48:725-730

7. Saari-Kemppainen A, Karjalainen O, Ylostalo P, Heinonen OP 1990 Ultrasound screening and perinatal mortality: controlled trial of systematic onestage screening in pregnancy. The Helsinki Ultrasound Trial. Lancet 336:387-391

8. Bricker L, Neilson JP 2000 Routine ultrasound in late pregnancy (after 24 weeks gestation). Cochrane Database Syst Rev CD001451.

9. Bricker L, Neilson JP 2000 Routine Doppler ultrasound in pregnancy. Cochrane Database Syst Rev CD001450.

\section{Pierre Gressens}

Inserm, U676, F-75019 Paris, France

Faculté de Médecine Denis Diderot

Université Paris 7, F-75019 Paris, France

Service de Neurologie Pédiatrique

Hôpital Robert Debré, F-75019 Paris, France

Petra S. Hüppi

Department of Pediatrics

Division of Child Development \& Growth

University of Geneva

1211 Geneva, Switzerland

Department of Neurology

Harvard Medical School

Children's Hospital

Boston, MA 02115

Received December 11, 2006; accepted

December 13, 2006.

Correspondence: Petra S. Huppi, M.D., University Children's Hospital Geneva, Division of Child Development \& Growth, Department of Pediatrics, 6 rue Willy Donzé, 1211 Geneva, Switzerland, Tél. 412238243 52, FAX 412238243 15; email:

Petra.Huppi@hcuge.ch

DOI: $10.1203 /$ pdr.0b013e318032252f 